Article

\title{
Network Traffic Features for Anomaly Detection in Specific Industrial Control System Network
}

\author{
Matti Mantere *, Mirko Sailio and Sami Noponen \\ VTT Technical Research Centre of Finland, Kaitovayla 1, Oulu 90571, Finland; \\ E-Mails: mirko.sailio@vtt.fi (M.S.); sami.noponen@vtt.fi (S.N.) \\ * Author to whom correspondence should be addressed; E-Mail: matti.mantere @ vtt.fi; \\ Tel: +358-40-570-8720.
}

Received: 27 June 2013; in revised form: 5 August 2013 / Accepted: 10 September 2013 /

Published: 25 September 2013

\begin{abstract}
The deterministic and restricted nature of industrial control system networks sets them apart from more open networks, such as local area networks in office environments. This improves the usability of network security, monitoring approaches that would be less feasible in more open environments. One of such approaches is machine learning based anomaly detection. Without proper customization for the special requirements of the industrial control system network environment, many existing anomaly or misuse detection systems will perform sub-optimally. A machine learning based approach could reduce the amount of manual customization required for different industrial control system networks. In this paper we analyze a possible set of features to be used in a machine learning based anomaly detection system in the real world industrial control system network environment under investigation. The network under investigation is represented by architectural drawing and results derived from network trace analysis. The network trace is captured from a live running industrial process control network and includes both control data and the data flowing between the control network and the office network. We limit the investigation to the IP traffic in the traces.
\end{abstract}

Keywords: industrial control systems; anomaly detection; machine learning; network security 


\section{Introduction}

Acquiring a commercial off-the-shelf (COTS) intrusion detection system (IDS) or network security monitor (NSM) set-up and placing it in an arbitrary location in an industrial control system (ICS) network is a straightforward task in itself. However, customization of the system and rewriting of the various rules is needed before meaningful functionality can be achieved [1]. Device manufacturers and service providers increasingly require remote connections to more of the devices in the field, and therefore industrial automation is slowly merging into the Internet of Things. Despite this, the ICS network environments are still very isolated compared to the traditional ICT organization's networks. Internet connections in the ICS environments should be managed and monitored carefully.

In the paper [2] we discussed the applicability of the machine learning approach to decrease the amount of manual customization required for deploying NSM system or IDSs in an ICS network.

After the initial research on the challenges and possibilities of this approach in [2] and sources explained in Section 2, we present a case study on the applicability of machine learning based approaches and possible feature selection for anomaly detection in the particular network environment under investigation. The intention is not to differentiate between generic or targeted attacks in the future, but is currently mainly targeted at more generic IP traffic anomaly detection. The paper is based on a conference paper [3].

The network traffic captured data on which we based the discussion on the applicability of the features was captured from a live factory environment and is studied in detail in the Section 3.1. The information concerning the nature and location of the industrial site in question as well as the automation equipment in use is withheld. This non-disclosure is due to the requirements by the parties responsible for the site's operation.

\section{Related Work}

Intrusion Detection Systems have been studied for over three decades, but using such systems in an ICS environment is a relatively new topic. The first study of an IDS that uses anomaly detection is presented by Denning [4] in 1986. Her model uses statistics for anomaly detection and can be seen as foundation for most anomaly based IDS deployments today. In addition, the network security monitoring models presented by Heberlein et al. [5] can be seen as foundation work for detecting anomalies in the network traffic. Garcia-Teodoro et al. [6] have reviewed several anomaly-based intrusion detection systems and present the challenges related to this method. According to them, the main challenge is is how to assess the method and how compare the performance with other approaches. Authors of [7] present the problematic of anomaly detection and state that most of the IDS deployments are still based on misuse detection. According to them, one of the main challenges is the diversity of network traffic in the Internet. Cheung et al. [8] introduced the idea of taking advantage of the regularity of ICS traffic by model-based anomaly detection. Their approach is on detecting zero-day attacks by model-based monitoring in Modbus TCP traffic. Hadeli et al. [9] have pointed out that the determinism of ICS traffic can be leveraged when configuring IDS rules. In [10], anomaly-based Intrusion Detection is used for detecting attacks in SCADA systems, and the authors note that insider attacks are harder to detect with this method. The authors of [11] use machine learning techniques on ICS environment 
intrusion detection with promising detection rates. They note that relevant future research subject is extracting significant features of the network traffic. Briesemeister et al. [12] have integrated anomaly detection with Security Information and Event Management (SIEM) which provides visualizations of critical infrastructure network attacks. Authors in [13] discuss in a more general context the sensor placement of intrusion detection systems.

An implementation of anomaly detection system using self-organizing maps algorithm (SOM) for anomaly detection is presented by Ramadas et al. in [14]. The source code of the system described, which we were provided with, also gave insight into a one way of implementing such a system. Currently, the most important aspect in the paper is the general description of the features selected for the algorithm and overall system and context. In [15] authors present a multilayer security architecture for anomaly detection in ICS networks. They use visualization tools for a better understanding of the results. Our method lacks visualization at the moment, but it is defined as an important goal in the later stages of our deployment. Morris et al. [16] use IDS rulesets for detecting anomalies in ICS protocols, but their solution does not apply machine learning techniques.

This article is an expanded version of an earlier workshop paper by the authors which was presented at the second Workshop on Security of Systems and Software Resiliency, Besancon, France, 2012, and published in its proceedings by IEEE Computer Society [3]. We have also discussed the intrusion detection challenges concerning machine learning in this context in paper [2].

\section{Materials and Methods}

The materials and methods used in this study are explained in this section. We use roughly the separation into three functional levels-device, cell and plants levels-that we also used previously in [2] as originally explained in [17].

On the device level the real-time requirements are very strict [18]. Typical devices doing the communicating are actuators, programmable logic controllers (PLC), sensors and such. Very little amounts of data are transmitted. Data transmitted represent two categories: data transfers. which are typically cyclic and periodic are one category, and alarms another. The data transfers are composed of control and measurement data that flows between field equipments. Alarms interrupt the normal data flow [17].

The cell level has the responsibility to coordinate various device controllers that reside in the device level. Communication typically consists of transmissions of tens of kilobytes in structured form in a client-server model [17].

The plant level interconnects the cell controllers to enterprise resource planning (ERP) systems. It does most of its communications during non-critical times transmitting much larger amounts of data compared to the previous two levels [17]. Networks present in a factory environment should also be segmented by use of firewalls and demilitarized zones (DMZ), terminating connections coming from outside of the ICS network at these DMZ's. This segmentation into security areas should not be confused with the above functional differentiation. The security segmentation and its particularities is discussed extensively in several research papers and other documents, such as [1]. The division into separated 
functional levels allows for the creation of separate models for anomaly detection. What is anomalous in one functional level can be normal when another level is concerned.

Due to the architecture of the network under scrutiny and its primary communication method, the functional layer separation is unclear. This will be discussed in Section 3.1.

\subsection{Network Structure and Available Traffic}

The target network is a ring topology ethernet network depicted in Figure 1. The network does not follow the recommended structure for demilitarized zones and separated communications, but does have an firewall between the ICS network and the office network. Multicast is used for most communications. The usage of multicast has a significant impact on the possible features to be used for the machine learning anomaly detection system.

The usage of the functional layers as presented in [17] for defining a separate set of features for each of the layers is not a straight-forward task in this environment. The use of multicast communication and ring topology network results in a situation where traffic from different functional levels is present in monitoring locations such as Tap1 and Tap2. For defining a separate set of features for each functional level the traffic in the network would have to be further analyzed and categorized, which would require considerable additional work.

Figure 1. Overview of the network structure of the factory site.

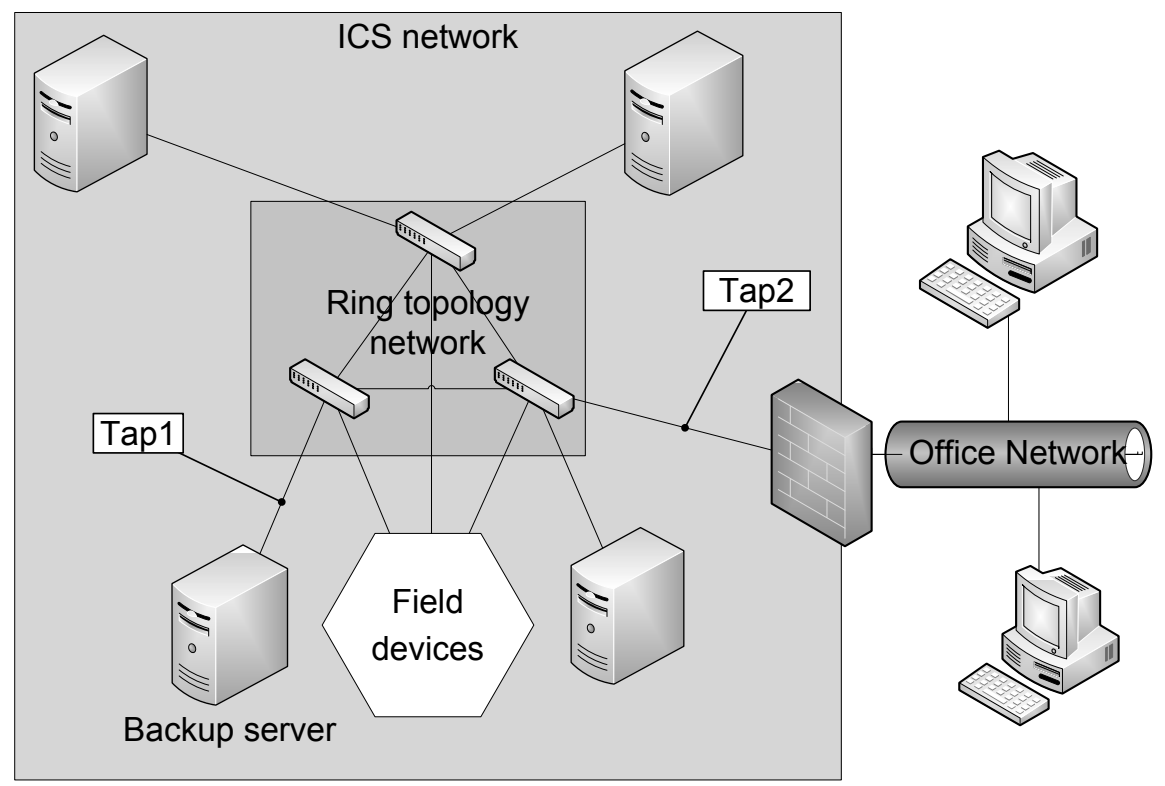

Available network traffic consists of traces captured from a live factory environment as well as traces captured from a laboratory environment with ICS equipment. The traces captured from the network are hour long and from two different locations: Tap1 and Tap2. Tap1 and Tap2 are within the ring topology ethernet network used mainly with multicast for control traffic. In the first capture, only traffic within the ICS network used for controlling the production systems and related traffic is visible. The location of this first capture is the Tap1 as depicted in Figure 1. For the Tap2, also depicted in Figure 1 we see 
the same multicast traffic as from Tap1 plus the non-multicast traffic to and from the office network that is separated from the control network via a firewall.

Traffic from Tap1 included a total of 3,168,660 packets. From Tap2 a total of 2,838,227 packets was recorded. Average packet size for Tap1 recorded traffic was 135.10 bytes with data byte rate of 109,257.90 B/s and for Tap2 134.23 B/s with data byte rate of 83,525.07 bytes/s. Packet capture duration was $3918 \mathrm{~s}$ for Tap2 and for Tap1 it was $4561 \mathrm{~s}$. This information is depicted in the Table 1. The capture files were also sliced into shorter segments for statistical analysis using editcap tool which is distributed with Wireshark [19]. Statistics of these slices is found in Table 2 and specifically for average packet slices in consecutive $600 \mathrm{~s}$ slices in Figure 2.

Table 1. Statistics of the packet captures, connection number without the RAID controller messages.

\begin{tabular}{ccc}
\hline Attribute & Capture location tap1 & Capture location tap2 \\
\hline Duration of capture & $3,918 \mathrm{~s}$ & $4,561 \mathrm{~s}$ \\
No. of packets & $3,168,660$ & $2,838,227$ \\
Avg. size of packets & $135.10 \mathrm{bytes}$ & $134.23 \mathrm{bytes}$ \\
Avg. data rate & $109,257.90 \mathrm{bytes} / \mathrm{s}$ & $83,525.07 \mathrm{bytes} / \mathrm{s}$ \\
Capture size & $478,792,912 \mathrm{bytes}$ & $426,382,869 \mathrm{bytes}$ \\
Avg. packet rate & 808.70 packets/s & 622.26 packets/s \\
No. of connections & 613 & 930 \\
No. of TCP connections & 8 & 174 \\
No. of UDP flows & 598 & 713 \\
No. of ICMP flow & 0 & 36 \\
Portion of multicast packets & $92.38 \%$ & $96.97 \%$ \\
\hline
\end{tabular}

The traffic included an amount of unencrypted control information for the system operators, visible in analysis by Tcpdump and Wireshark. This information included names of various systems, commands available for their operation as well as other information regarding their type. The traffic seen at the Tap2 includes traffic of all the three levels described in Section 3.

A single device, a Promise RAID controller caused noise in the traffic. All of the traffic directed to port 712 was of this type. Of the total of 3,168,660 packets that made up the traffic captured from Tap1 2610 packets consisted of the Promise RAID controller messages with the following nearly static part: PROMISE_RAID\#KBOI\#Hello World!

These messages made up a disproportionately large part of the connection log produced by the Bro NSM, of the total of 3216 connections logged 2610 were actually single instances of these messages, each of which registered as a new connection. 
Table 2. Statistics of the $600 \mathrm{~s}$ interval slices of the packet captures.

\begin{tabular}{ccccc}
\hline \multirow{2}{*}{ Tap } & Data byte rate (B/s) & Avg. packet size(B) & Avg. packet rate \\
\hline \multirow{2}{*}{$0 s$} & Tap1: & $110,764.06$ & 135.12 & 819.73 \\
& Tap2: & $81,069.95$ & 132.19 & 613.29 \\
\hline \multirow{2}{*}{600 s } & Tap1: & $112,781.12$ & 135.16 & 834.73 \\
& Tap2: & $81,336.13$ & 132.15 & 615.48 \\
\hline \multirow{2}{*}{1200 s } & Tap1: & $109,876.51$ & 135.06 & 813.55 \\
& Tap2: & $87,409.34$ & 138.72 & 630.12 \\
\hline \multirow{2}{*}{1800 s } & Tap1: & $108,285.54$ & 135.09 & 801.58 \\
& Tap2: & $83,917.03$ & 133.33 & 629.39 \\
\hline \multirow{2}{*}{2400 s } & Tap1: & $107,414.70$ & 135.09 & 795.11 \\
& Tap2: & $85,160.06$ & 133.65 & 637.20 \\
\hline \multirow{2}{*}{3000 s } & Tap1: & $107,521.36$ & 135.07 & 796.06 \\
& Tap2: & $83,499.59$ & 133.01 & 627.75 \\
\hline \multirow{2}{*}{3600 s s } & Tap1: & $107,203.58$ & 135.14 & 793.27 \\
& Tap2: & $80,870.29$ & 132.31 & 611.23 \\
\hline \multirow{2}{*}{4200 s } & Tap1: & - & - & - \\
& Tap2: & $85,885.34$ & 141.26 & 608.00 \\
\hline \multirow{2}{*}{} & & & &
\end{tabular}

Figure 2. Average packet sizes for the 600 s slices of packet capture from Tap1 and Tap2.
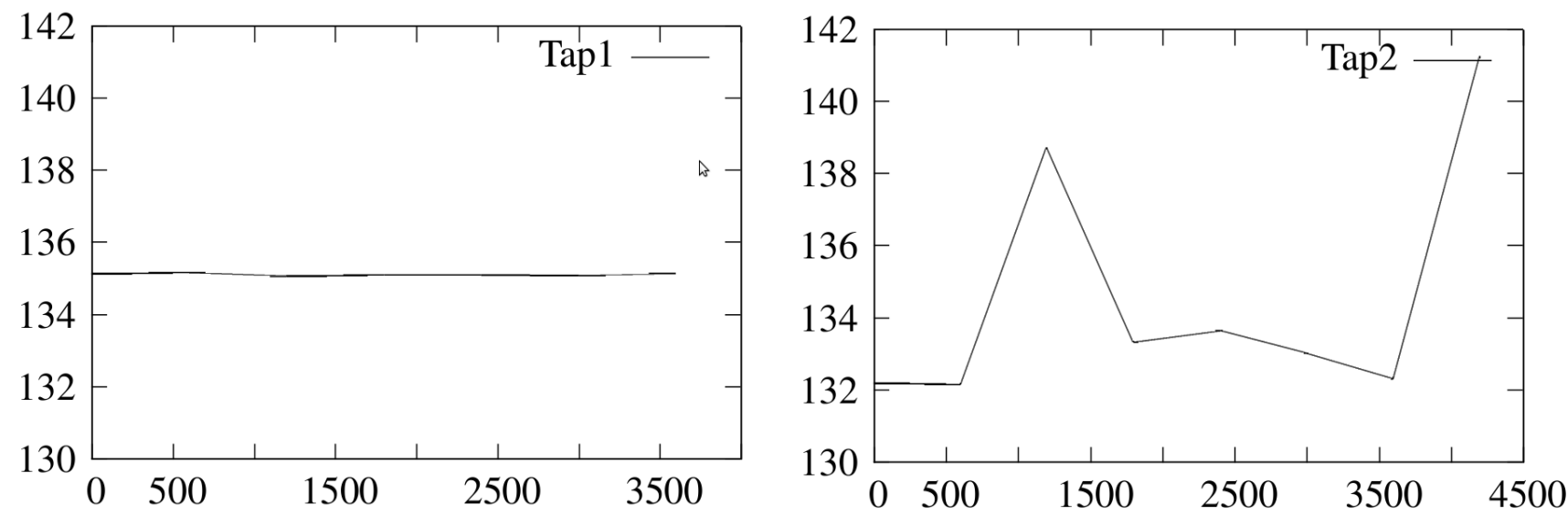

For the traffic recorded from Tap2, the total number of packets was 2,838,227 of which 3031 packets represented RAID controller traffic. 3223 connections were reported by Bro NSM of which again 3031 were RAID messages. For the duration of the packet capture and the location of the Tap $2,0.1 \%$ of the packets found in the network represented this type of unneeded traffic.

At a first glance, using Wireshark [19], some very suspicious protocols were reported by the tool, such as eDonkey, a peer-to-peer protocol. However, after a short investigation, it was revealed that these very sporadic and sparsely appearing protocols were also in fact messages by Promise RAID controllers present in the network. This particular behavior by Promise RAID controllers turned out to be causing the 
false appearance of a number different protocols in Wireshark analysis. After weeding out the protocols caused by RAID controller messages, the list of protocols presented in Table 1 remained.

No traffic in the network traces was found which would have been readily identified as being of a malicious origin. Hence all the traffic analyzed was assumed to be of benign origin, even if unnecessary or posing a risk. This assumption is critical for selection of possible features.

\subsubsection{Protocols Presentation}

The protocols listed in Table 3 were found to be present in the network traffic. From some of the protocols several different versions were seen, such as was the case with IGMP. The data displayed a diversity in versions, a need for which was not immediately clear. For example the IGMP protocol traffic includes both versions 1 and 2. This coincides with our initial expectation that systems such as this that have been modified over a period of time include redundant versions of protocols that are either forgotten to be present in the network or kept for compatibility purposes due to old and non-updated systems.

Table 3. Protocols seen in the traffic captures.

\begin{tabular}{ll}
\hline ARP & NBSS NetBIOS session service \\
\hline CDP Cisco Discovery Protocol & NDPS Novell Distributed Print System \\
DCERPC & NetBIOS Name Service \\
DCOM OXID Resolver & NTP Network Time Protocol \\
DHCP & PARLAY General Inter-ORB \\
GIOP General Inter-ORB Protocol & RMI Java \\
HP Switch Protocol & RELOAD \\
HTTP & SMB \\
ICAP Internet Content Adaptation Protocol & SMB NETLOGON \\
ICMP & SNMP \\
IGMP & SSDP \\
Intel ANS Probe & STUN Session Traversal Utilities for NAT \\
ISystemActivator & STP \\
LANMAN Remote Api & TCP \\
LLDP Link Layer Discovery Protocol & TPNCP TrunkPack Network Control Protocol \\
LLMNR Link-Local Multicast Name Resolution & UDP \\
Microsoft Windows Browser Protocol & VRRP Virtual Router Redundancy Protocol \\
MySQL & X11 \\
NBNS & \\
\hline
\end{tabular}

\subsection{Tools}

For the analysis of the example traces in this paper we used tcpdump [20], Wireshark [19] and its associated tools, Bro Network Security Monitor (Bro) [21,22] version 2.1. During the initial investigations Netmate and NetAI as bundled in [23] were used as well. 
Netmate and NetAI were used to calculate flow statistics from the traffic captures to aid in the initial analysis of the packet capture data. At a later stage Bro was mostly used for generating the connection information and the usage of Netmate and NetAI was dropped.

Tcpdump was used to capture and manipulate the traffic from the test network depicted in Figure 1. As the monitoring setup produced various packet capture files, we merged them in a bi-directional traffic file.

Wireshark, tcpdump and the included tools were used for calculating the statistics depicted in Table 2, Table 1 and Figure 2 and deriving the protocol list in Table 3. Wireshark and Tcpdump were also both used to investigate trace contents manually. This revealed additional information to the automatic analysis e.g. transmission of control information in plain text format.

Bro was used mainly to produce connection, domain name system and weird traffic logs in its network traffic analysis framework capability. Both the connection and domain name system logs proved very informative and would possibly be useful even without the added configuration of site-specific security policies written in the provided scripting language. The number of connections in Table 1 reflect Bro interpreting UDP and ICMP flows as pseudo-connections, based on its own internal logic [22].

The weird traffic log produced by Bro informed that the communication was triggering weird behavior events and would benefit from a closer look. The triggered weird events were of the following types listed below. Their cause and effect warrant further investigation into the network and its functionality, but is not in the scope of this paper. The events were:

$$
\text { non_IPv4_packet }
$$

above_hole_data_without_any_acks

possible_split_routing

DNS_label_len_gt_pkt
truncated_IP

connection_originator_SYN_ack

data_before_established

DNS_truncated_ans_too_short

\subsection{Features}

In the paper [2] a selection of 12 features useful for machine learning approach to network security monitoring in ICS network were discussed.

We reviewed these potential features against the findings of the network traffic analysis with mixed results. Some of the features were applicable for this particular network, some decisively not, whilst the feasibility of some remained uncertain. We chose to split the longer packet captures into $600 \mathrm{~s}$ slices for simple statistical analysis. The selection of exactly $600 \mathrm{~s}$ was arbitrary, and other intervals could have been chosen.

\subsubsection{Throughput}

Feature feasibility: Stability of throughput is typical for ICS networks. Changes in throughput can be used to detect high traffic events (scanning, DoS, malfunction). Attacks with low traffic requirements are not detectable with monitoring throughput.

Our data: The average packet rates and sizes in both recording locations are depicted in Table 2. From these it appears that there is some fluctuation in both locations within the total recording interval. 
Longer period of traffic recordings could provide us with further insight, but from this data it is difficult to assess whether the average packet rate would be usable.

\subsubsection{IP Address-Port Pairs}

Feature feasibility: In ICS networks that use static IP allocation, IP address—port pairs are expected to remain constant: appearance of new IP-port pair indicates a new service being started on the system containing the IP.

Our data: The initial and most simple features that would be usable are the address pairs of connections or packets consisting of source and destination internet protocol addresses and ports. This is simple to achieve and in a static environment would quickly bring to fore any sudden new addresses appearing.

Strong multicast environment poses real challenges for using IP - port pairs, as the multicast protocol obscures the destination data. However even in this environment, tracking IP - port pairs highlights the atypical unicast flows.

\subsubsection{Average Size of Packets}

Feature feasibility: Average size of packets is another good network behavior indicator. Many device level systems with high real time requirements send packets without extra buffering creating distinct average size statistics for these networks. In many networks the average size of packets can be considered to be a good indicator of normal behavior.

Our data: Average size of the packets looks like a feasible feature in the context and time frame of the packet captures. When the trace files where split into $600 \mathrm{~s}$ slices and further analyzed, it was clear that there was minimal fluctuation in the Tap1 recording which was not exposed to traffic coming and going to the office side. In the Table 2 we can see that the Tap2 has a wider fluctuation in the average packet size and data rate. Average packet size over the total duration of both packet capture files can be found in Table 1 Should the stability of the average packet size persist for longer periods of time as well during nominal operation of the ICS network, we argue that either increases or decreases in this value could signal an anomalous situation. Average packet sizes are visualized in Figure 2 which clearly shows the difference in the variance of average packet sizes.

\subsubsection{Timing}

Feature feasibility: Packet timing and interval between packets from a network node is meaningful data for many ICS networks. The strict real time requirements of the system, especially in the device level of the network, make the timing statistics of the traffic stand out from typical application traffic.

Our data: We analyzed packet intervals and timing of packets. The packet intervals had great variation and showed no simple patterns or regularity. No patterns or useful behavior was detected in packet timing. Analysis of the most active network nodes showed no exploitable characteristics in individual systems packet intervals. Further analysis is required for usability of timing features, especially for single network nodes. 


\subsubsection{Flow Direction}

Feature feasibility: Flow direction indicates which system initiates the connection. For typical operation, the flow is initiated by one system, requiring a service, after which the amount of data sent from one system to the other is likely to be predictable, especially when the service in question is known. Variance in this behavior indicates abnormal behavior.

Our data: In our samples, the usage of a multicast protocol makes it impossible to group messages to "conversations" as with unicast protocols. The destination data is a multicast address, not an IP address of a system.

\subsubsection{Flow Duration}

Feature feasibility: The duration of flow is typically the TCP session length. It represents the amount of time, that a system or service requires to conduct it's network functions with it's destination system. As the number of services in industrial network nodes is limited, the duration of flows is deemed to have little variance.

Our data: With multicast protocol, the flow duration could not be meaningfully used, and is not a plausible detection feature in the network under scrutiny.

\subsubsection{Payload Form}

Feature feasibility: Payload of packets for applications in a ICS environment are often strictly defined. The changes in payload form indicates a change in system behavior.

Our data: Analysis on the feasibility of payload form require further work.

\subsubsection{Payload Data}

Feature feasibility: Payload data can be used to detect misconfigured systems or malicious actions. Payload abnormalities are a good sign for detection. The usage of default user credentials should be avoided in an ICS network. This enables the detection of systems trying out default credentials as a first line of attack. Detection also exposes poorly configured legitimate systems.

Our data: Simple string detection discovered no flagrant default usage of most typical usernames and passwords in our traffic, but more work is required for a meaningful assessment of the feature. Analysis however detected both confidential data and data useful for attacker for identifying both network and SCADA hardware.

\subsubsection{MAC—IP Mapping}

Feature feasibility: MAC to IP mapping can be used in any LAN to detect changes in hardware components. The appearance of new MAC indicates that new hardware has appeared in the network. While MAC addresses can be forged, they are still usable for impersonation detection. It also helps the ICS network operator to be aware of all legitimate hardware in the system.

Our data: No MAC to IP mapping anomalies were detected in the traffic. 


\subsubsection{Network Protocol}

Feature feasibility: The protocols used in the ICS network should be strictly defined and limited. Appearance of new protocols in the traffic indicate serious change in the network. If the networking protocol is identified using only port number, as is typical with Wireshark, the detection may be incorrect. Many systems use non-standard port addresses to conduct their network activity.

Our data: Network protocol using port numbers was not a feasible feature in this environment. The Promise RAID controller messages render it difficult to use as explained earlier in Section 3.1. More advanced protocol identification might change the feature to be highly usable.

\subsubsection{Protocol Settings}

Feature feasibility: The protocols settings used in ICS networks are typically static, selected to guarantee the best performance of the ICS network. Monitoring the protocol settings of used protocols will detect misconfigured services and malicious activities.

Our data: The feasibility of protocol settings as a feature requires further research.

\subsubsection{Connectivity Number}

Feature feasibility: The number of connections for different systems is very static in most ICS networks. This depends widely on the role of the node.

Our data: Connectivity numbers are not usable in the network under scrutiny as the usage of multicast obscures the destination of data.

\section{Results}

The analysis of packet captures showed, as expected, that not all of the traffic found in the ICS network in question is strictly necessary. This complies with the initial hypothesis. The situation could be improved by renewing the equipment and following stricter policies when deploying new devices with network access. Several versions of protocols exist and totally useless traffic is present, such as the "Hello World!" messages sent by the RAID controller with poor configuration. Some of the older protocol versions might even potentially pose security risks. However, it must be noted that in a factory environment the overhaul process of network equipment is a more complicated task than in the typical office environment.

The features that were investigated against the results of the network trace analysis did show that some of the initially thought features could have been useful, while some would definitely not have worked in this particular environment. Interestingly the average packet size in the factory side of the network appeared the most promising, with very little variance present. When the longer trace was split in to $600 \mathrm{~s}$ intervals and analyzed, the average packet size appeared nearly constant 135 bytes.

Our work continues with machine learning approach for anomaly detection in the context of ICS and restricted networks. Our current early implementation prototype is based on SOM [24] approach built as an extension for Bro [22]. It is implemented using Bro's built-in scripting language and currently includes connection and network state based information for forming the feature vectors in the SOM 
lattice. Work on this extension module continues and the feature set is evolving and expanding as the investigation and implementation progresses. Presenting the system and testing it with real traffic data with and without attacks or similar anomalies will be discussed in other papers at a later stage.

The investigations described in this paper were important on the path to develop the prototype implementation.

\section{Discussion}

The difficulties in deployment of intrusion detection and network monitoring systems for ICS networks are further compounded by the redundancies and unnecessary traffic in the network. In this type of environment the amount of unnecessary traffic should be minimized. The analyzed data was not free of unneeded traffic, but was relatively stable of the short period of time we had recorded. The short period of recording time of one hour is an issue which needs to be remedied in the future. Longer packets captures and larger traces are needed to more accurately assess the possible anomaly detection approaches and the applicability of various features that could be used if a machine learning approach would be used. Deployment process of a network monitoring or intrusion detection system would be beneficial also in a way of bringing attention to the issue of noise, obsolete protocols and unneeded traffic possibly present in ICS networks.

The same capturing device was used for both locations, with the traffic captures therefore being sequential to each others, this creates an unknown level of uncertainty as to whether some of the differences in traffic attributes perceived actually resulted from time dependent changes in the network environment. This was due to the restricted access to the industrial site in question. This restriction caused an uncertainty which warrants further investigation into the issue.

The features discussed earlier in [2] were partially applicable and we will continue to design and implement a proof-of-concept system. Further investigation with more extensive packet captures from the target network would be required to implement a system for this particular environment. After a proof-of-concept has been implemented and validated for a single environment, extending the approach to other networks with a wider optional feature base can be considered.

\section{Acknowledgements}

We would like to thank our industrial collaborators for providing us the necessary access to record the network traffic under scrutiny in this article as well as information concerning the nature of the industrial site. This paper is based on research work done in projects called DIAMONDS [25], funded by TEKES and INCYSE, funded by VTT.

\section{Conflicts of Interest}

The authors are not party to any conflict of interest concerning the work presented in this paper. 


\section{References}

1. The U.S. Department of Homeland Security (DHS) and Division's Control Systems Security Program (CSSP). Common Cybersecurity Vulnerabilities in Industrial Control Systems; National Cyber Security Division: Washington, DC, USA, 2011.

2. Mantere, M.; Uusitalo, I.; Sailio, M.; Noponen, S. Challenges of Machine Learning Based Monitoring for Industrial Control System Networks. In Proceedings of the 26th International Conference on Advanced Information Networking and Applications Workshops, Fukuoka, Japan, 26-29 March 2012; pp. 968-972.

3. Mantere, M.; Sailio, M.; Noponen, S. Feature Selection for Machine Learning Based Anomaly Detection in Industrial Control System Networks. In Proceedings of the 2nd Workshop on Security of Systems and Software Resiliency, IEEE Computer Society, Besancon, France, 20-23 November 2012; pp. 771-774.

4. Denning, D. An intrusion-detection model. IEEE Trans. Softw. Eng. 1987, SE-13, 222-232.

5. Heberlein, L.; Dias, G.; Levitt, K.; Mukherjee, B.; Wood, J.; Wolber, D. A Network Security Monitor. In IEEE Computer Society Symposium on Research in Security and Privacy 1990; IEEE Computer Society: Oakland, CA, USA, 1990; pp. 296-304.

6. Garca-Teodoro, P.; Daz-Verdejo, J.; Maci-Fernndez, G.; Vazquez, E. Anomaly-based network intrusion detection: Techniques, systems and challenges. Comput. Secur. 2009, 28, 18-28.

7. Sommer, R.; Paxson, V. Outside the Closed World: On Using Machine Learning for Network Intrusion Detection. In Proceedings of the 2010 IEEE Symposium on Security and Privacy (SP), Oakland, CA, USA, 16-19 May 2010; pp. 305-316.

8. Cheung, S.; Dutertre, B.; Fong, M.; Lindqvist, U.; Skinner, K.; Valdes, A. Using Model-based Intrusion Detection for SCADA Networks. In Proceedings of the SCADA Security Scientific Symposium, Miami, FL, USA, 24-25 January 2007.

9. Hadeli, H.; Schierholz, R.; Braendle, M.; Tuduce, C. Leveraging Determinism in Industrial Control Systems for Advanced Anomaly Detection and Reliable Security Configuration. In Proceedings of the IEEE Conference on Emerging Technologies and Factory Automation (ETFA 2009), Mallorca, 22-25 September 2009; pp.1-8.

10. Yang, D.; Usynin, A.; Hines, J.W. Anomaly-Based Intrusion Detection for SCADA Systems. In Proceedings of the 5th International Topical Meeting on Nuclear Plant Instrumentation, Control and Human Machine Interface Technologies (NPIC\&HMIT 05), Albuquerque, NM, USA, 12-16 November 2006.

11. Linda, O.; Vollmer, T.; Manic, M. Neural Network Based Intrusion Detection System for Critical Infrastructures. In Proceedings of International Joint Conference on Neural Networks, Atlanta, GA, USA, 14-19 June 2009.

12. Briesemeister, L.; Cheung, S.; Lindqvist, U.; Valdes, A. Detection, Correlation, and Visualization of Attacks Against Critical Infrastructure Systems. In Proceedings of the 2010 Eighth Annual International Conference on Privacy Security and Trust (PST), Ottawa, Canada, 17-19 August 2010. 
13. Chen, H.; Clark, J.; Shaikh, S.; Chivers, H.; Nobles, P. Optimising IDS Sensor Placement. In Proceedings of the ARES 10 International Conference on Availability, Reliability, and Security, Krakow, Poland, 15-18 February 2010; pp. 315-320.

14. Ramadas, M.; Ostermann, S.; Tjaden, B. Detecting Anomalous Network Traffic with Self-Organizing Maps. In Proceedings of the Sixth International Symposium on Recent Advances in Intrusion Detection, LNCS, Pittsburgh, PA, USA, 8-10 September 2003; Springer Verlag: Berlin, Germany, 2003; pp. 36-54.

15. Valdes, A.; Cheung, S. Intrusion Monitoring in Process Control Systems. In Proceedings of the 42nd Annual Hawaii International Conference on System Sciences HICSS, Big Island, HI, USA, 5-8 January 2009; pp. 1-7.

16. Morris, T.; Vaughn, R.; Dandass, Y. A Retrofit Intrusion Detection System for MODBUS RTU and ASCII Industrial Control Systems. In Proceedings of the 45th Hawaii International Conference on System Sciences, Maui, HI, USA, 4-7 January 2010.

17. Bertoluzzo, M.; Buja, G.; Vitturi, S. Ethernet Networks for Factory Automation. In Proceedings of the 2002 IEEE International Symposium on Industrial Electronics ISIE 2002, L'Aquila, Italy, 8-11 July 2002; Volume 1, pp. 175-180.

18. Gaderer, G.; Sauter, T.; Ring, F.; Nagy, A. A Novel, Wireless Sensor/actuator Network for the Factory Floor. In Proceedings of the 2010 IEEE Sensors, Waikoloa, HI, USA, 1-4 November 2010; pp. 940-945.

19. Wireshark Protocol Analyzer. Available online: http://http://www.wireshark.org/ (accessed on 30 January 2013).

20. Tcpdump. Available online: http://http://www.tcpdump.org/ (accessed on 30 January 2013).

21. Bro Network Security Monitor. Available online: http://www.bro-ids.org/ (accessed on 30 January 2013).

22. Paxson, V. Bro: A system for detecting network intruders in real-time. Comput. Netw. 1999, 31, 2435-2463.

23. NetMate-flowcalc. Available online: http://dan.arndt.ca/projects/netmate-flowcalc/ (accessed on 26 June 2013).

24. Kohonen, T. Self-Organizing Maps, 3rd ed.; Springer-Verlag Inc.: New York, NY, USA, 2001.

25. DIAMONDS Project. Available online: http://www.itea2-diamonds.org/index.html (accessed on 23 September 2013).

(c) 2013 by the authors; licensee MDPI, Basel, Switzerland. This article is an open access article distributed under the terms and conditions of the Creative Commons Attribution license (http://creativecommons.org/licenses/by/3.0/). 